Roberto Gerson de Albuquerque Azevedo

Suporte ao controle e à apresentação de objetos de mídia tridimensionais em NCL

Dissertação de Mestrado

Dissertação apresentada como requisito parcial para obtenção do grau de Mestre pelo Programa de PósGraduação em Informática do Departamento de Informática da PUC-Rio.

Orientador: Prof. Luiz Fernando Gomes Soares 
Roberto Gerson de Albuquerque Azevedo

\section{Suporte ao controle e à apresentação de objetos de mídia tridimensionais em NCL}

Dissertação apresentada como requisito parcial para obtenção do grau de Mestre pelo Programa de PósGraduação em Informática do Departamento de Informática do Centro Técnico e Científico da PUC-Rio. Aprovada pela Comissão Examinadora abaixo assinada.

Prof. Luiz Fernando Gomes Soares Orientador Departamento de Informática - PUC-Rio

Prof. Alberto Barbosa Raposo Departamento de Informática - PUC-Rio

Prof. Renato Fontoura de Gusmão Cerqueira Departamento de Informática - PUC-Rio

Prof. José Eugenio Leal Coordenador(a) Setorial do Centro Técnico Científico - PUC-Rio 
Todos os direitos reservados. É proibida a reprodução total ou parcial do trabalho sem autorização da universidade, do autor e do orientador.

\section{Roberto Gerson de Albuquerque Azevedo}

Recebeu seu título de Bacharel em Ciências da Computação pela Universidade Federal do Maranhão em 2008. Atualmente integra o grupo de pesquisadores do Laboratório Telemídia, desenvolvendo pesquisas na área de Sistemas Hipermídia e TV Digital.

Ficha Catalográfica

Azevedo, Roberto Gerson de Albuquerque

Suporte ao controle e à apresentação de objetos de mídia tridimensionais em NCL/ Roberto Gerson de Albuquerque Azevedo; orientador: Luiz Fernando Gomes Soares. - 2010.

113 f. : il. ; $30 \mathrm{~cm}$

Dissertação (mestrado) - Pontifícia Universidade Católica do Rio de Janeiro, Rio de Janeiro, 2010.

Incluí referências bibliográficas.

1. Informática - Teses. 2. Sistemas Hipermídia. 3. Linguagens declarativas 3D. 4. Nested Context Model. 5. Nested Context Language. 6. Grafo de Cena. 7. Grafo de Rotas. I. Soares, Luiz Fernando Gomes. II. Pontifícia Universidade Católica do Rio de Janeiro. Departamento de Informática. III. Título. 
Para os meus pais e meus grandes mestres, Tarcísio e Carminda, por todo o amor, apoio e dedicação. 


\section{Agradecimentos}

Muitas pessoas estiveram presentes na minha vida durante esses dois anos de curso, sem as quais este trabalho não seria possível. Sendo assim, deixo aqui meus humildes agradecimentos a todos que direta ou diretamente contribuíram com este trabalho. Em especial, agradeço:

Ao Professor Luiz Fernando Gomes Soares, meu orientador, pelo aprendizado contínuo que é trabalhar ao seu lado.

A todos os amigos do laboratório Telemídia: Alexandra, Álvaro, Bruno, Carlos de Salles, Carlos Eduardo, Eduardo, Felipe Nagato, Felipe Nogueira, Francisco, Guilherme, José Geraldo, Luciana Rosa, Marcelo Moreno, Márcio Moreno, Rafael Savignon, Ricardo, Romualdo e Vinicius; pelo ótimo ambiente de trabalho que propiciam e por estarem sempre à disposição para discutir as mais inusitadas ideias.

Aos pesquisadores que aqui estiveram no passado, os quais criaram os alicerces para os trabalhos hoje desenvolvidos, inclusive este.

Aos meus irmãos, Tarcísio Filho, Tiago Henrique e Maria Virgínia, pela compreensão da minha ausência.

A minha namorada, Vanessa, pelo apoio incondicional e por estar sempre me motivando.

Aos meus pais Tarcísio Braga e Virgínia Carminda, pela referência que são na minha vida, pela educação que me propociaram e pelo amor absoluto.

E, finalmente, a todos os colegas, professores e funcionários do Departamento de Informática da PUC-Rio. 


\section{Resumo}

Azevedo, Roberto Gerson de Albuquerque; Soares, Luiz F. G. Suporte ao controle e à apresentação de objetos de mídia tridimensionais em NCL. Rio de Janeiro, RJ, Brasil, 2010. 113p. Dissertação de Mestrado Departamento de Informática, Pontifícia Universidade Católica do Rio de Janeiro,

O mundo em que vivemos é formado por um espaço fisicamente tridimensional e é natural que o ser humano busque representá-lo da forma mais fiel possível, também em ambientes virtuais. Na Web, por exemplo, já existem vários esforços na busca do suporte a criação de aplicações 3D interativas de forma declarativa, grande parte deles são baseados nos conceitos de grafos de cena e grafos de rotas. Grafos de cena têm se tornado um padrão de modelagem espacial de aplicações tridimensionais por meio de uma abordagem hierárquica e declarativa. Por sua vez, o comportamento do grafo de cena usualmente tem sido especificado por meio de grafos de rotas ou linguagens imperativas. No que se refere à TV Digital Interativa (TVDi), por outro lado, ainda há muito a ser feito. Nested Context Language (NCL) é a linguagem declarativa padrão para o sistema de TV Digital Terrestre ISDB-T B $_{B}$ e Recomendação ITU-T para serviços IPTV, que permite a autoria de documentos hipermídia por meio de uma abordagem simples e expressiva. Embora NCL não restrinja qualquer tipo de objeto de mídia, na sua versão atual (3.0), ela trata apenas objetos de mídia bidimensionais, relacionando-os temporal e espacialmente. Dada a importância de NCL no cenário de TVDi, esta pesquisa objetiva discutir como tal linguagem pode também controlar objetos tridimensionais, permitindo a criação de aplicações 3D para TVDi. Como caso especial, este trabalho discute como NCL pode controlar o comportamento de objetos 3D compostos representados por grafos de cenas, discutindo suas vantagens e desvantagens em relação ao uso de grafos de rotas. Visando testar a proposta deste trabalho, foi adicionado um exibidor de mídia X3D (linguagem baseada em grafo de cena) à implementação de referência do Ginga-NCL, responsável por executar aplicações NCL. Adicionalmente, também é proposta a extensão dos eventos tratados por NCL, para refletir eventos específicos de ambientes 3D, e a incorporação de regiões, baseadas em objetos geométricos 3D, à NCL, com o objetivo de possibilitar a apresentação de objetos de mídia 2D sobre a superfície de objetos 3D. 


\section{Palavras-chave}

NCL; Aplicações 3D; Televisão Digital Interativa; Ginga-NCL; Grafos de Cena; X3D. 


\section{Abstract}

Azevedo, Roberto Gerson de Albuquerque. Soares, Luiz F. G. (Advisor). Supporting three-dimensional media object control and presentation in NCL. Rio de Janeiro, RJ, Brasil, 2010. 113p. MSc . Dissertation Departamento de Informática, Pontifícia Universidade Católica do Rio de Janeiro.

The world where we live in is physically formed by a three-dimensional space and it is natural human being wants to represent it as faithful as possible. In Web for example there are a lot of efforts in order to support the creation of interactive 3D applications, most of them based on scene graphs and route graphs. Scene graphs have become a standard for modeling spatial 3D applications from a hierarchical and declarative approach. In order to represent the scene graphs behavior, route graphs or imperative languages are more commonly used. As regards Interactive Digital Television (iDTV), on the other hand, there is still a lot of work to be done. Nested Context Language (NCL) is the standard declarative language for Terrestrial Digital Television Systems (ISDB-T) and IPTV (ITU-T), which allows the hypermedia document authoring through a simple and expressiveness approach. Even though NCL does not restrict any type of media object, in its current version (3.0), NCL treats only two-dimensional objects, relating them temporally and spatially. Given the importance of NCL in the iDTV scenario, this research aims discuss how it can also control three-dimensional objects. As a special case, this work discusses how NCL can control composite object behavior represented by scene graphs, discussing its advantages and disadvantages. In order to test what was proposed, an X3D (which is an ISO standard language based on scene graphs) player was incorporated to the GingaNCL reference implementation, which is responsible to play NCL applications. Additionally, it is also proposed a new set of NCL events, in order to reflect threedimensional events, and the incorporation of NCL regions based on threedimensional geometric, so that the presentation of two-dimensional media objects over 3D object's surfaces is possible.

\section{Keywords}

NCL; 3D applications; Interactive digital television; Ginga-NCL; Scene graphs; X3D. 


\section{Sumário}

1 Introdução 16

$\begin{array}{ll}\text { 1.1. Objetivos e Contribuições } & 18\end{array}$

1.2. Organização da Dissertação 19

2 Conceitos Básicos $\quad 21$

2.1. Grafo de Cena 21

2.1.1. Otimizações para grafos de cena 24

2.2. Grafo de Rotas 26

2.3. NCL 28

2.3.1. Nested Context Model 28

$\begin{array}{ll}\text { 2.3.2. Nested Context Language } & 31\end{array}$

3 Trabalhos Relacionados 36

3.1. Linguagens baseadas em Grafos de Cena 36

3.1.1. Extensible 3D (X3D) Graphics 37

3.1.2. Extensible MPEG-4 Textual Format (XMT) 41

3.1.3. XMT e NCL 45

3.2. Ginga-NCL 46

4 Objetos de mídia tridimensionais em NCL 50

4.1. Objetos de mídia 3D declarativos como objetos de mídia em documentos NCL 52

4.1.1. Definição de Âncoras de Conteúdo em Grafos de Cena 53

4.1.2. Definição de Âncoras de Propriedades em Grafos de Cena 55

4.1.3. Eventos NCL para Ambientes 3D 57

4.1.4. Parametrização de eventos NCL 60

4.1.5. Relacionamento entre eventos em objetos 3D e objetos de mídia NCL 62

4.2. Controlando o Comportamento de Grafos de Cena por meio 
$\begin{array}{ll}\text { de NCL } & 68\end{array}$

4.2.1. Elos e conectores NCL em documentos X3D 70

4.3. Comparativo entre grafo de rotas, XMT e NCL 71

5 Estendendo as regiões da NCL 3.0 75

5.1. Atributos de dimensão e posicionamento em NCL 3.0

5.2. Transformações em regiões NCL 78

5.2.1. Transformações 2D em regiões NCL 81

5.2.2. Transformações 3D em regiões NCL 86

5.3. Definição de regiões NCL como superfície de objetos 3D 91

6 Implementação 95

6.1. Exibidor X3D no Ginga-NCL 96

$\begin{array}{lr}\text { 6.1.1. Modelagem } & 97\end{array}$

6.1.2. FreeWRL 100

6.2. Implementação de Referência do Ginga-NCL baseada em SDL e OpenGL 102

6.2.1. Configuração dos Componentes do Ginga-NCL para utilizar SDL e OpenGL como backend 105

7 Conclusões e Trabalhos Futuros 107

7.1. Contribuições da Dissertação 108

$\begin{array}{ll}\text { 7.2. Trabalhos Futuros } & 108\end{array}$

8 Referências Bibliográficas $\quad 110$ 


\section{Lista de figuras}

Figura 1 Modelo de um grafo de cena baseado em DAG.

Figura 2 Grafos de cena modelados sem considerar a

posição espacial (a) e considerando a posição espacial (b) dos seus nós.

Figura 3 Exemplo de um grafo de rotas.

Figura 4 Máquina de estados de eventos NCM.

Fonte: (SOARES e RODRIGUES, 2005)

Figura 5 Nós, elos e nós de composição.

Fonte: (SOARES NETO, SOARES, et al., 2010) 31

Figura 6 Exemplo de código NCL que apresenta um vídeo em tela cheia. $\quad 33$

Figura 7 Exemplo de elo (relacionamento) e conector (relação) em NCL. $\quad 34$

Figura 8 Exemplo de um Grafo de Cena X3D. 38

Figura 9 Classic VRML e XML Encoding de um mesmo modelo X3D. 39

Figura 10 Modelo da Figura 9 em execução $\quad 40$

Figura 11 Perfis X3D. Fonte: (BRUTZMAN e DAILY, 2007). 40

Figura 12 Exemplo dos principais componentes da arquitetura

de um exibidor X3D. Adaptado de: (DALY e BRUTZMAN, 2008). 41

Figura 13 Exemplo de um documento XMT- $\Omega$

Figura 14 Exemplo de código XMT- $\Omega$ demonstrando evento

de colisão entre objetos 3D.

Figura 15 Diferença entre modelagem baseada em grafo de cena (a)

e XMT- $\Omega$ (b), ao entrelaçar conteúdo e comportamento.

Figura 16 Arquitetura do Ginga-NCL e Ginga-CC (Common Core)

Fonte: (MORENO, 2010) 47

Figura 17 NCL como linguagem de cola, incluindo objetos de mídia 3D. 52

Figura 18 Definição de âncora de conteúdo em objetos de mídia

definidos por grafo de cena, por meio do atributo label. 54

Figura 19 Definição de âncora temporal em NCL por meio do atributo clip. $\quad 55$

Figura 20 Definição de uma âncora temporal no grafo de cena. 55

Figura 21 Mapeamento de âncoras de propriedade ao embutir objetos

de mídia NCL. 
Figura 22 Definição de âncoras de propriedades em grafos de cena.

Figura 23 Exemplo de um conector causal em NCL.

Figura 24 Exemplo de um conector causal utilizando nome de papéis

reservados em NCL. 58

Figura 25 Possível trajeto de um usuário ao interagir com uma aplicação. $\quad 60$

Figura 26 Exemplo de parâmetros de eventos. 61

Figura 27 Exemplo de conector parametrizado e elo utilizando esse conector 62

Figura 28 Visão estrutural do Exemplo 1.

Figura 29 Código fonte do objeto de mídia X3D do Exemplo 1.

Figura 30 Código fonte NCL do Exemplo 1.

Figura 31 Visão estrutural do Exemplo 2. 64

Figura 32 Código fonte do objeto de mídia X3D do Exemplo 2.

Figura 33 Código fonte NCL do Exemplo 2.

Figura 34 Visão estrutural do Exemplo 3.

Figura 35 Código fonte do objeto de mídia X3D do Exemplo 3.

Figura 36 Código fonte NCL do Exemplo 3. 67

Figura 37 Exemplo de animação utilizando apenas X3D (grafo de rotas). $\quad 68$

Figura 38 Exemplo de relacionamento seqüencial entre duas mídias. 69

Figura 39 Exemplo de código fonte NCL que pode representar o

relacionamento sequencial do exemplo da Figura 38. 70

Figura 40 Exemplo de documento X3D com nós e elos NCL. 71

Figura 41 Exemplo de código XMT onde a semântica do contêiner

temporal <par> pode ser ignorada para executar mídias em sequência.

Figura 42 Definição de atributos de dimesão e posicionamento dos

objetos de mídia por meio de: (a) regiões, (b) descritores e

(c) propriedades de objetos de mídia.

Figura 43 Atributos de posicionamento e dimensão de regiões na

versão 3.0 de NCL. Fonte: (SOARES NETO, SOARES, et al., 2010).

Figura 44 Definição de transformações em regiões, descritores e

propriedades de objetos de mídia em NCL através do parâmetro

transform - notação (i).

Figura 45 Definição de transformações em regiões, descritores e propriedades de objetos de mídia em NCL, por meio de atributos 
específicos para cada transformação - notação (ii).

Figura 46 Sistema de coordenadas 2D em regiões NCL.

Tabela 4 Novos atributos para rotação, cisalhamento e espelhamento, seguindo a proposta (ii).

Figura 47 Rotação de região NCL especificada no elemento <region> segundo a notação (i) e (ii).

Figura 48 Exemplo de definição de transformação de cisalhamento

em NCL, por meio de parâmetros nos descritores, segundo as notações (i) e (ii).

Figura 49 Demonstração da transformação de flipHorizontal e flipVertical em imagem.

Figura 50 Exemplo de definição de espelhamento vertical e horizontal segundo as notações (i) e (ii).

Figura 51 Sistema de coordenadas cartesianas local de uma região (segundo regra da mão direita) tomado como base para transformações tridimensionais.

Figura 52 Exemplo de translação de uma região de exibição de um objeto de mídia especificada no elemento <region> NCL.

Figura 53 Parâmetros para a definição de uma rotação em uma região NCL. $\quad 90$

Figura 54 Região NCL com rotação de 90 graus ao redor do eixo Y.

Figura 55 Definição de regiões NCL como superfície de objetos 3D por meio do atributo geometry.

Figura 56 Especificação de uma região NCL como a superfície de objetos geométricos reutilizando os módulos de geometria de X3D.

Figura 57 Arquitetura da Implementação de Referência do

Ginga-NCL evidenciando os pontos que foram modificados por esta

dissertação. Adaptado de (MORENO, 2010).

Figura 58 Diagrama de Classe Exibidor X3D adicionado à

Implementação de Referência do Ginga-NCL.

Figura 59 Diagrama de Classe Adaptador X3D adicionado à Implementação de Referência do Ginga-NCL.

Figura 60 Geração de nós de sensores automaticamente pelo X3DPlayer.

Figura 61 Diagrama de classe dos novos eventos adicionados 
nesta dissertação.

Figura 62 Diagrama de classes evidenciando a interface de eventos

do usuário, disponível pela Implementação de Referência,

e as novas implementações dessas interfaces baseadas em SDL.

Figura 63 Diagrama de classes evidenciando a interface responsável

por abstrair as funções da tela do dispositivo e implementação em SDL.

Figura 64 Diagrama de classe evidenciando a implementação de superfícies de renderização em SDL.

Figura 65 Exemplo da definição dos novos componentes baseados

em SDL no modelo de componentes da Implementação de Referência. 


\section{Lista de tabelas}

Tabela 1 Novos eventos NCL, específicos de objetos de mídia 3D.

Tabela 2 Tabela comparativa entre XMT, Grafo de Rotas (X3D)

e o modelo NCM para o controle do comportamento de cenas 3D.

Tabela 3 Novas transformações 2D para rotação, cisalhamento e espelhamento, seguindo a proposta (i). 82

Tabela 5 Novas transformações 3D, seguindo a proposta de notação (i).

Tabela 6 Novos atributos para transformações 3D, seguindo a proposta de notação (ii). 